\title{
Positive effect of ozonotherapy on serum concentration of soluble interleukin-2 receptor and neopterin in patients with systemic sclerosis
}

\author{
Danuta Nowicka \\ Department of Dermatology, Venereology and Allergology, Wroclaw Medical University, Wroclaw, Poland \\ Adv Dermatol Allergol 2019; XXXVI (2): 158-163 \\ DOI: https://doi.org/10.5114/ada.2019.83651
}

\begin{abstract}
Introduction: Systemic sclerosis (SSc) is a chronic systemic autoimmune disease of unknown aetiology. No curative therapy exists, thus management includes controlling of symptoms and prevention of complications. Ozonotherapy has multiple beneficial therapeutic effects which can translate into improving prognostic factors for SSc.

Aim: To evaluate the effect of ozone on selected factors of inflammation: serum concentration of the soluble receptor of interleukin-2 (IL-2 SR), neopterin as well as the peripheral blood smear in patients with SSc.

Material and methods: Forty-two patients with SSc underwent two series of 10-minute baths in water with a mixture of air and ozone separated by a 10-day period without treatment. Examinations were performed at baseline and 10 days after completing the last treatment.

Results: The mean concentration of IL-2 sR decreased significantly from 1563.73 to $1249.86 \mathrm{pg} / \mathrm{ml}$. The mean concentration of neopterin decreased significantly from 12.06 to $10.9 \mathrm{nmol} / \mathrm{ml}$. Absolute monocytosis decreased insignificantly from 1.694 to $1.480 \mathrm{G} / \mathrm{l}$. Correlations between the disease duration and concentration of IL-2 sR were weak and negative, while between the disease duration and concentration of neopterin were weak and positive. Conclusions: Ozonotherapy had a beneficial influence on the immune response in patients with scleroderma. Balneotherapy may be considered as an additional therapeutic option, but further research is required.
\end{abstract}

Key words: ozone therapy, systemic sclerosis, cellular response, balneotherapy.

\section{Introduction}

Scleroderma, also known as systemic sclerosis, is a chronic systemic autoimmune disease of unknown aetiology. Its prevalence is higher in the USA (276/million in 1990) and Australia (233/million in 1999) while lower in Japan and Europe (France: 158/million in 2001 and England: $88 /$ million in 2000) [1]. It is estimated that in Poland scleroderma affects about 10,000 people [2]

Therapies offered to patients suffering from scleroderma have limited efficacy and yet, the curative treatment has not been developed. Management of the disease consists of controlling of symptoms and preventing of complications [3-5]. Ozone therapy is not considered the recommended treatment for systemic sclerosis, but it has multiple beneficial therapeutic effects $[6,7]$. Ozone is one of natural gases composed of three atoms of oxygen. Ozone as a strong oxidizer can stimulate the cellular antioxidant enzymes. Its positive effects were observed in complementary treatment of hypoxic and ischemic syndromes such as wound healing including a diabetic foot [8]. Ozone can also exert systemic effects observed in diabetes. It can be helpful in treatment of chronic pulmonary diseases, and lumbar-herniated disc. It is also well known in dental medicine [7]. The main interest of the study was whether the stimulation of antioxidative responses can translate into improving prognostic factors for systemic sclerosis.

Monitoring of the disease is challenging due to the complex pathogenesis [9-11]. Our study was based on the measurement of the two compounds. The concentration of the soluble receptor of interleukin 2 (IL-2 SR) is dependent upon the activation of cellular immunologic response and considered a prognostic indicator in many diseases including systemic sclerosis [12-16]. The physiological role of neopterin has not yet been fully elucidated; however, the synthesis of neopterin is stimulated by the cytokines, and thus, its increased concentration has been

Address for correspondence: Danuta Nowicka MD, PhD, Department of Dermatology, Wroclaw Medical University, 1 Chalubinskiego St, 50-368 Wroclaw, Poland, phone: +48 7178423 35, fax: +48 71 3270942, e-mail: danuta.nowicka@umed.wroc.pl Received: 13.09.2017, accepted: 13.12.2017. 
used as a marker to monitor diseases whose pathogenesis involves immune responses [17, 18].

\section{Aim}

The aim of the study was to evaluate the effect of ozone on selected factors for inflammation: the concentration of the soluble receptor IL-2, neopterin as well as the picture of peripheral blood in patients with systemic sclerosis.

\section{Material and methods}

The study included 42 patients with systemic sclerosis with the age ranging from 32 to 73 years (average: $45 \pm 12.7)-28$ women and 14 men, hospitalised in the university clinic between 2003 and 2006. The diagnosis of systemic sclerosis (SSc) was based, according to ACR/ EULAR recommendations, on clinical presentation, laboratory results, imaging, functional evaluation of internal organs and examination of capillaries (capillaroscopy). Rodnan score was measured at baseline and after the treatment. The duration of scleroderma was on average $6.55 \pm 5.18$ years, ranging from 12 months to 23 years. The patients participating in this study were not subjected to other therapeutic methods at the moment of enrolment; they discontinued treatment at least 1 month before entering the study for reasons unrelated to the present study.

In the present study, two series of baths separated by ten days without treatment were conducted. One series lasted 10 days and consisted of 10-minute baths daily. The patients were exposed to the mixture of air and ozone dissolved in water in the amount of 4000 mg/treatment. The laboratory examination was carried out before the treatments and 10 days after the last treatment. For baths, the rehabilitation equipment Ozonomatic Jolly Med (Ozonomatic srl, Rome, Italy) was used [19, 20].

The concentration of IL-2 sR was measured with immune-enzymatic method ELISA using the Quantikine Human IL-2 sRo Immunoassay kit (R\&D Systems, McKinley Place, MN, USA). To assess the concentration of neopterin, the Neopterin ELISA test no. RE59321 (IBL, Hamburg, Germany) was employed. The morphological analysis of peripheral blood was carried out using the haematological analyser K 4500 (Sysmex, Kobe, Japan). The concentration of haemoglobin, haematocrit, the number of white blood cells, red blood cells and blood platelets were measured.

Blood smears, after drying, were evaluated using the May-Grünwald-Giemsa method. In the preparation, 100 successive cells of the white blood cell system were analysed. The absolute number of monocytes in $\mathrm{G} / \mathrm{l}$, which was taken into account in further research, was calculated from the proportion of monocytes.

The study was approved by the Institutional Review Board and was conducted in accordance with the Declaration of Helsinki. All subjects gave informed consent to participation in the study.

\section{Statistical analysis}

Statistical analysis was carried out with the Statistica software v. 10 (StatSoft, Tulsa, OK, USA). To determine the statistical significance of differences between two groups, Student's t-test was used. To evaluate relationships, the Pearson product-moment correlation coefficient was applied. Data were considered to be statistically significant at a value of $p<0.05$.

\section{Results}

After two series of ten 10-minute comprehensive baths in water with the mixture of air and ozone, the baseline concentration of IL-2 SR and neopterin decreased significantly. The baseline value of absolute monocytosis exceeded normal values defined as $<1 \mathrm{G} / \mathrm{l}$ with the percentage of monocytes in peripheral blood $<9 \%$. In the studied group, the percentage of monocytes ranged between 5 and 40\% (mean: $25.42 \pm 3.4$ ) at baseline and their absolute number ranged from 0.225 to $4.290 \mathrm{G} / \mathrm{l}$ (mean: $1.694 \pm 0.64)$. The increased values of absolute monocytosis $>1 \mathrm{G} / \mathrm{l}$ was observed in $61 \%$ of patients. After the treatment, the percentage of monocytes ranged between 6 and 40\% (mean: $19.76 \pm 4.1$ ) and the absolute value decreased insignificantly. The comparison of selected parameters before and after ozone therapy is presented in Table 1.

Correlations between duration of the disease and values of IL-2 SR and neopterin were weak both before and after the treatment (Figure 1). In patients with shorter

Table 1. Comparison of selected parameters before and after two series of ozone therapy

\begin{tabular}{lccccc}
\hline Variable & \multicolumn{2}{c}{$\begin{array}{c}\text { Before ozone therapy } \\
(n=42)\end{array}$} & \multicolumn{2}{c}{$\begin{array}{c}\text { After ozone therapy } \\
(n=42)\end{array}$} \\
\cline { 2 - 5 } & Mean \pm SD & Range & Mean \pm SD & Range & \\
\hline Concentration IL-2 sR [pg/ml] & $1563.73 \pm 714$ & $845.0-3394$ & $1249.86 \pm 86$ & $675.1-3135.1$ & 0.02 \\
\hline Neopterin concentration [nmol/l] & $12.06 \pm 2.3$ & $8-18.5$ & $10.9 \pm 2.1$ & $7.5-17$ & 0.012 \\
\hline Absolute monocytosis [G/l] & $1.694 \pm 0.640$ & $0.225-4.290$ & $1.480 \pm 0.212$ & $0.168-3.120$ & 0.2 \\
\hline
\end{tabular}



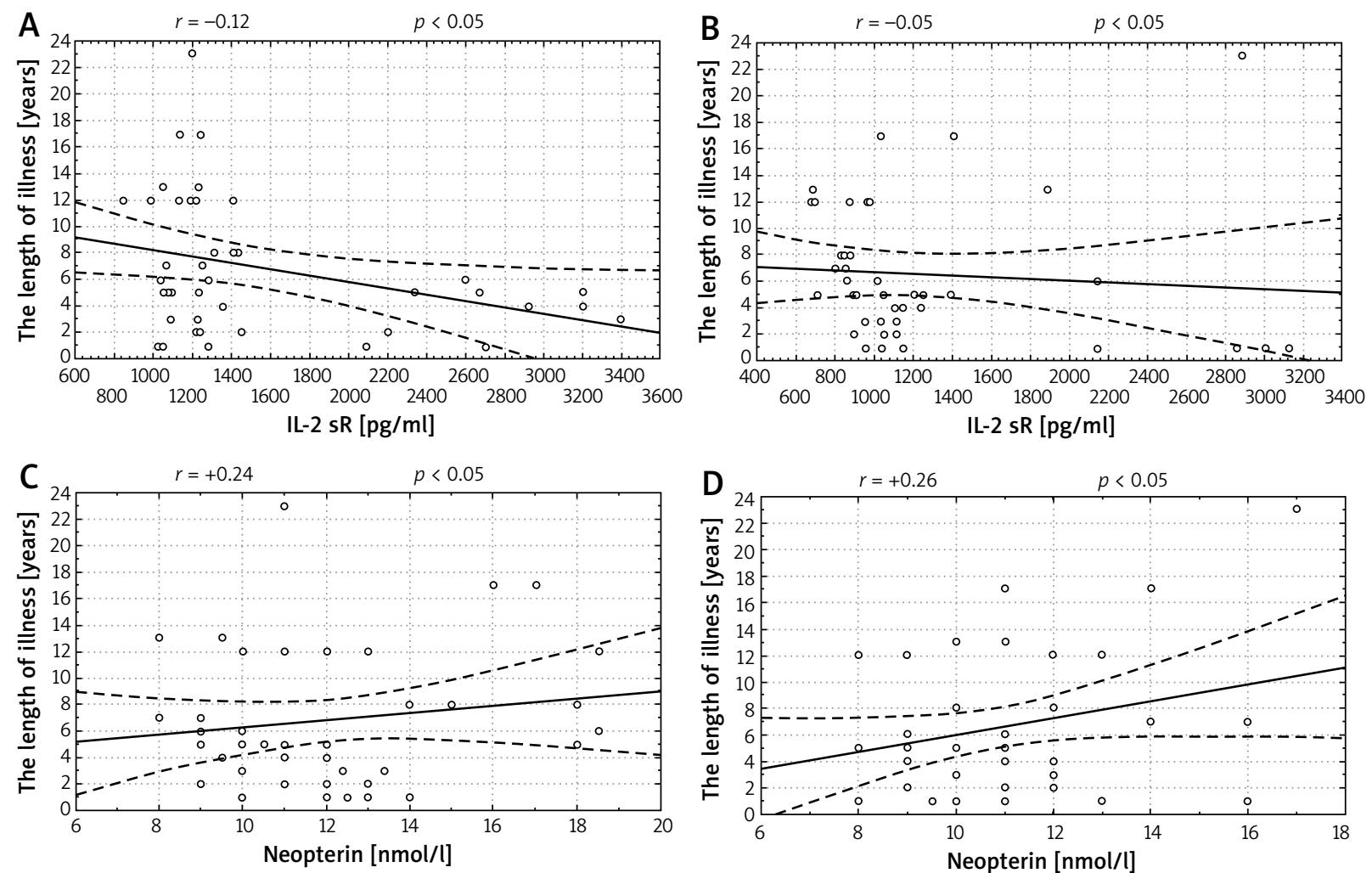

Figure 1. Correlations between the length of illness and concentration of soluble receptor IL-2 SR and neopterin before and after the treatment. A - Correlation between the length of illness and concentration of soluble receptor IL-2 sR $(r=-0.12)$ at baseline, B - correlation between the length of illness and concentration of IL-2 SR $(r=-0.05)$ after a series of treatments, $\mathbf{C}$ - correlation between the length of the illness and concentration of neopterin $(r=+0.24)$ at baseline, D - correlation between the length of the illness and concentration of neopterin $(r=+0.26)$ after two series of treatment

Table 2. Comparison of correlations between selected parameters

\begin{tabular}{lcc}
\hline Correlation analysis & Correlation coefficient $r$ & Correlation direction and level \\
\hline Length of illness - IL-2 sR before ozonotherapy & -0.12 & Negative, weak \\
\hline Length of illness - IL-2 sR after ozonotherapy & -0.05 & Negative, faint \\
\hline The length of illness - neopterin before ozonotherapy & +0.24 & Positive, weak \\
\hline The length of illness - neopterin before ozonotherapy & +0.26 & Positive, weak \\
\hline IL-2 sR - neopterin & +0.16 & Positive, weak \\
\hline
\end{tabular}

illness, the activity of the inflammatory state was higher and the factors in this process were more susceptible to the activity of anti-inflammatory substances. The positive correlation between the length of the disease and concentration of neopterin proves that it can be used as a marker of disease progress.

Considering that both the in-vivo production of IL-2 and neopterin depend on the synthesis of IFN- $\gamma$, the correlation between those two markers of the inflammatory state was analysed. The positive correlation between the concentration of IL-2 SR and neopterin confirms the common mechanism of releasing cytokine and solid protein. Correlation coefficients are presented in Table 2.
Clinical examination showed a decrease in skin thickness. The mean baseline Rodnan score was $42.0 \pm 3.5$ (range: 33-48 scores) and after the ozone treatment dropped to $27.3 \pm 5.27$ (range: $19-38$ scores). Statistical analysis revealed that this difference was significant $(p=0.004)$.

No side effects were reported by patients.

\section{Discussion}

Our study shows that in response to ozonotherapy, the concentration of IL-2 SR and neopterin decreased significantly in the peripheral blood. Also the absolute number of monocytes decreased, though insignificantly. The changes in immune response markers were associ- 
ated with the reduction in patients' skin thickness as measured with Rodnan score.

Neopterin is a protein whose production results from the activation of the immune system and can be considered a marker of the activation of the cellular type response [18]. IL-2 is a cytokine produced by the Th1 lymphocytes responsible for intensifying the disease process, and the more sensitive marker of this process is the concentration of its soluble receptor [12, 15, 16, 21]. The significant decrease in both the neopterin and IL-2 sR was observed after ozone therapy, which suggests that ozonotherapy may silence over-stimulated immune response.

Monocytosis is the most characteristic deviation occurring in the hematopoietic system in connective tissue diseases. Activation of monocytes in scleroderma, in addition to their increased number, is indicated by the presence of numerous vacuoles in the cytoplasm of these cells. The results of the present study are in line with the thesis. The present study showed increased monocytosis at baseline, but it dropped insignificantly after the treatment with ozone.

The immune processes mediated by the activated T-lymphocytes, monocytes, granulocytes, the decrease in NK-cell activity and increased production of immunoglobulins by B-lymphocytes becomes the basis for the vascular changes and the disability in internal organ functions which in the course of systemic sclerosis is the secondary process. The T-lymphocytes are responsible for cellular response as well as collaboration with the B-lymphocytes in the course of humoral immunity. The activated T-lymphocytes produce a number of markers of inflammatory state activation: pro-inflammatory cytokines such as IL-2, IL-2 sR, and anti-inflammatory ones such as IL-4, IL-6, IL-10 and SCD4. In the present study, it is essential to understand the role of the first population because of their participation in the production of the inflammatory state factors which take part in the etiopathogenesis of scleroderma: IL-2 and interferon $\gamma$ (IFN- $\gamma$ ) which stimulates macrophages to produce neopterin [14, 22].

Currently to assess the progress of scleroderma, sensitive markers of inflammation are used. The concentration of IL-2 and IL-2 SR are measured most often as markers of activity of T-lymphocytes. In the present study, a decrease in IL-2 SR concentration was found, which confirms that IL-2 participates in the aetiology of the illness. It is produced by the stimulated T-lymphocytes as a cytokine which is a key factor in immune system activation. Our results are in line with Lis et al. outcomes who found that IL-2 is a more sensitive indicator than IL-6 which is present in a lower percentage of patients with systemic sclerosis [14, 22].

The negative correlation between the concentration of IL-2 SR and the length of illness proves that the biggest activation of cell response occurs at the onset of illness and during its progress - when the secondary participa- tion of immune humoral response components and the fading of the active fibrotic process occur, the concentration of IL-2 falls insignificantly, but, most often exceeds the normal range.

In the present study, the concentration of IL-2 sR was higher in comparison with the reference values in $72 \%$ of patients. Data from literature suggest that higher values are presented by $20-80 \%$ of patients; however, an unchanged level of this cytokine does not exclude the activation of immune system cells. One of the reasons for this may be the flaw of the laboratory method used.

In the present study, the concentration of IL-2 SR significantly decreased after ozonotherapy. The obtained decrease was lower than in Lis team's study on combined treatment with immunosuppressive medicines cyclophosphamide and prednisolone [22]. It should be stressed that the present experiment pertained to the assessment of how the substances, which are not typical immunosuppressants, act and that it was carried out over a much shorter period of time.

In order to assess the intensity of the inflammatory state, neopterin was additionally used. The production of neopterin is a consequence of macrophage activation by the factor produced by the stimulated T-lymphocytes, most of all by IFN- $\gamma$, which was shown in in-vitro research. IFN- $\gamma$ stimulates the synthesis of neopterin in concentrations 400 times stronger than IFN- $\alpha$ [17]. In the experiment, an increase in concentration of neopterin in $81 \%$ of the patients was observed indicating the presence of macrophage activation and an intensified cell response in the etiopathogenesis of systemic sclerosis, which is in line with other reports from the literature [23].

After application of two series of baths, it was shown that there was a significant decrease in neopterin concentration in patients' serum. This corresponds to the results obtained after immunosuppressive treatment of cancer patients [15].

A positive correlation between the concentration of IL-2 SR and neopterin shows that there is a mutual interdependency between these indicators. The activated T-lymphocytes produce IL-2 and IFN- $\gamma$ and cytokines stimulating the macrophages to produce neopterin. This process is confirmed by other authors who report a positive correlation between the concentration of neopterin and IL-2 SR in patients suffering from silicosis - the illness which together with systemic sclerosis - is the illness of connective tissue [13]. The decrease in the concentration of IL-2 SR and neopterin resulting from the applied balneotherapy and ozonotherapy indicates the beneficial influence of ozone on the pathomechanism of immune changes in scleroderma, thus these indicators can be used as markers of illness progression.

In the present study, hemogram was also assessed showing the increased value of absolute monocytosis. Monocytes are present in peripheral blood and penetrate into tissues where they function as macrophages. 
Despite the fact that they are very important for the immune system, express many receptors on their surface, and produce many compounds such as enzymes (the synthase of nitric oxide - NOS), complement components, angiogenetic factors, bindings proteins, active lipids, chemokines and growth factors such as erythropoietin, IFN- $\alpha$, IFN- $\gamma$, IL-1, 3, $6,8,10,12$, M-CSF, PDGF, TNF, they are not taken into account during the study of pathomechanisms of many illnesses which proceed with an increase in their number and activity. In the literature, there are only few studies on the influence of monocytosis, particularly on the role of the factor which is chemotactic for monocytes (monocyte chemotactic protein 1 - MCP-1) produced in the course of scleroderma. This protein is also produced by the T-lymphocytes, fibroblasts, endothelial cells, neutrophils as well as keratinocytes. In the quoted research, the increased concentration of MCP-1 released, among others, under the influence of platelet-derived growth factor (PDGF) was shown $[3,24]$.

All the mechanisms of cellular response disorders causing a chronic T-lymphocytes activation which leads to the chronic inflammatory process become the main reason for the fibrosis determining the clinical changes in the course of scleroderma. The limitation of the activation of cellular response, which requires thorough knowledge of the mechanisms which regulate it, has thus become the challenge in modern therapy of sclerosis-related illnesses [25].

In the present study, the control group of healthy subjects was not included, which does not allow for comparison of the effect of ozone on biomarker concentration in healthy people. However, the concentration of neopterin in healthy subjects is considerably lower than in patients with chronic diseases. Data from the large studies suggests that the concentration of neopterin is approximately $5 \mathrm{nmol} / \mathrm{l}$ in healthy subjects while in the present study it was more than 2-fold higher [26, 27]. Similarly, the plasma concentration of IL-2 SR in healthy subjects was reported to be lower than in the present study. The normal range of IL-2 SR is considered to be below 790 pg/ $\mathrm{ml}$, while in the present study, it was more than 2-fold higher before the treatment [13]. The comparison with healthy subjects does not bring any information about changes in the clinical condition characteristic for systemic sclerosis.

\section{Conclusions}

Outcomes of the present research confirm the usefulness of balneotherapy and ozonotherapy in systemic sclerosis. Ozonotherapy may be an effective method of slowing the progression of disease. It may be used alone or with pharmacotherapy in mild forms or during remissions. Additionally, this method is safe and cheap.

\section{Conflict of interest}

The author declares no conflict of interest.

\section{References}

1. Chifflot H, Fautrel B, Sordet C, et al. Incidence and prevalence of systemic sclerosis: a systematic literature review. Semin Arthritis Rheum 2008; 37: 223-35.

2. Kanecki K, Goryński P, Tarka P, et al. Incidence and prevalence of systemic sclerosis (SSc) in Poland - differences between rural and urban regions. Ann Agric Environ Med 2017; 24: 240-4.

3. Tsifetaki N, Botzoris V, Alamanos Y, et al. Bosentan for digital ulcers in patients with systemic sclerosis: a prospective 3-year follow-up study. J Rheumatol 2009; 36: 1550-2.

4. Manno R, Boin F. Immunotherapy of systemic sclerosis. Immunotherapy 2010; 2: 863-78.

5. Hinchcliff $M$, Varga J. Systemic sclerosis/scleroderma: a treatable multisystem disease. Am Fam Physician 2008; 78: 961-8.

6. Re L, Rowen R, Travagli V. Ozone therapy and its use in medicine. Cardiology 2016; 134: 99-100.

7. Elvis AM, Ekta JS. Ozone therapy: a clinical review. J Nat Sci Biol Med 2011; 2: 66-70.

8. Martinez-Sanchez G, Al-Dalain SM, Menendez S, et al. Therapeutic efficacy of ozone in patients with diabetic foot. Eur J Pharmacol 2005; 523: 151-61.

9. Banica L, Besliu A, Pistol G, et al. Quantification and molecular characterization of regulatory T cells in connective tissue diseases. Autoimmunity 2009; 42: 41-9.

10. Besliu AN, Banica LM, Lonescu R, et al. Role of cellular immunity in systemic sclerosis pathogenesis: update on CD4+T cells population studies. Roum Arch Microbiol Immunol 2009; 68: 5-13.

11. Choi JJ, Min DJ, Cho ML, et al. Elevated vascular endothelial growth factor in systemic sclerosis. J Rheumatol 2003; 30: 1529-33.

12. Vettori S, Maresca L, Cuomo G, et al. Clinical and subclinical atherosclerosis in systemic sclerosis: consequences of previous corticosteroid treatment. Scand I Rheumatol 2010; 39: 485-9.

13. Hayashi H, Maeda M, Murakami S, et al. Soluble interleukin-2 receptor as an indicator of immunological disturbance found in silicosis patients. Int I Immunopathol Pharmacol 2009; 22: 53-62.

14. Krause S, Beyerlein A, Winkler C, et al. Soluble interleukin-2 receptor alpha in preclinical type 1 diabetes. Acta Diabetol 2014; 51: 517-8.

15. Gonda K, Shibata M, Shimura T, et al. Serum soluble interleukin-2 receptor is increased in malnourished and immunosuppressed patients with gastric and colorectal cancer: possible influence of myeloid-derived suppressor cells. World J Oncol 2012; 3: 158-64.

16. Tsukamoto S, Ishikawa S, Yamauchi A, et al. Serum soluble interleukin-2 receptor levels in patients with renal cell carcinoma: a comparison of values before and after surgery. Hinyokika Kiyo 2000; 46: 695-9.

17. Melichar B, Spisarová M, Bartoušková M, et al. Neopterin as a biomarker of immune response in cancer patients. Ann Transl Med 2017; 5: 280.

18. Muller MM, Curtius HC, Herold M, et al. Neopterin in clinical practice. Clin Chim Acta 1991; 201: 1-16.

19. Antoszewski Z, Kulej J, Wygledowski M, et al. Some aspects of ozone therapy. Przegl Lek 1997; 54: 561-4. 
20. Antoszewski L, Moszkowicz T, Kozakiewicz J. Ozonotherapy in Polish medicine. Terapia 1996; 4: 22-8.

21. Chibowska M, Krasowska D, Weglarz J. Internal organ lesions in acrosclerosis patients: interleukin-2 and soluble receptor for interleukin-2 plasma levels. J Eur Acad Dermatol Venereol 2000; 14: 234-6.

22. Lis A, Brzezinska-Wcislo L, Wcislo-Dziadecka D. The evaluation of soluble interleukin-2 receptor levels in the serum before and after the immunosuppressive treatment in patients with systemic screrosis. Derm Klin 2006; 8: 165-9.

23. Goerdt S, Politz O, Schledzewski K, et al. Alternative versus classical activation of macrophages. Pathobiology 1999; 67: 222-6.

24. Goldstein BD. Cellular effects of ozone. Rev Environ Health 1977; 2: 177-202.

25. Koenig M, Dieude M, Senecal JL. Predictive value of antinuclear autoantibodies: the lessons of the systemic sclerosis autoantibodies. Autoimmun Rev 2008; 7: 588-93.

26. Cesur S, Aslan T, Hoca NT, et al. Clinical importance of serum neopterin level in patients with pulmonary tuberculosis. Int J Mycobacteriol 2014; 3: 5-8.

27. Hamerlinck FF. Neopterin: a review. Exp Dermatol 1999; 8: 167-76. 